\title{
Evolución de la formación profesional en España: medio siglo de cambios y reformas
}

\begin{abstract}
Vallejo, Mónica ${ }^{1}$
Molina-Saorín, Jesús ${ }^{2}$

Martínez-López, María José ${ }^{3}$

\section{Abstracto}

El objetivo de este artículo es describir el recorrido y evolución de la Formación Profesional en España a través de la normativa promulgada hasta el momento (leyes educativas y reales decretos). Concretamente son analizadas la Ley General de Educación (LGE), la Ley Orgánica de Ordenación General del Sistema Educativo (LOGSE), la Ley Orgánica de Calidad de la Educación (LOCE), Ley Orgánica de Educación (LOE) y la estrenada Ley Orgánica para la Mejora de la Calidad Educativa (LOMCE). En ellas se examina su contenido a través de tres grandes bloques: estructura y currículum establecido para estas enseñanzas, profesorado y condiciones profesionales y; por último, un apartado de consideraciones generales, en las que se realiza una valoración de las mejoras y críticas de la ley con respecto a la pasada. Las conclusiones alcanzadas detallan cómo se han ido construyendo las enseñanzas de Formación Profesional, al tiempo que permite valorar cuál ha sido su impacto real (respecto a su finalidad), así como los interrogantes y debilidades de la normativa educativa promulgada a lo largo de estos últimos 45 años. La lectura de este trabajo pretende facilitar la reflexión sobre las luces y sombras que aún aqueja la Formación Profesional en este país; siendo este un momento importante, tras la reciente implantación de la Formación Profesional Básica.
\end{abstract}

\section{Formación Profissional. Médio Siglo. Reformas}

\section{Resumo}

O objetivo deste artigo é descrever o percurso e evolução da Formação Profissional na Espanha através da normativa promulgada até o momento (leis educativas e reais decretos). São analisadas, especificamente, a Lei Geral de Educação (LGE), a Lei Orgânica de Classificação Geral do Sistema Educativo (LOGSE), a Lei Orgânica de Qualidade da Educação (LOCE), Lei Orgânica de Educação (LOE) e a recente Lei Orgânica para a Melhora da Qualidade Educativa (LOMCE). Nelas examinase o conteúdo através de três grandes blocos: estrutura e currículum estabelecido para estes ensinos, professorado e condições profissionais; e, por último, um apartado de as considerações gerais nas quais realiza-se uma avaliação das melhoras e críticas da lei com respeito à legislação passada. As conclusões atingidas detalham como foram sendo construindo os ensinos de Formação Profissional, ao mesmo tempo em que permite valorizar qual tem sido seu impacto real (com respeito à sua finalidade), bem como as interrogações e debilidades do regulamento educativo promulgado ao longo dos últimos 45 anos. A leitura deste trabalho pretende facilitar a reflexão sobre as luzes

\footnotetext{
1 Profesor Titular de Universidad, en el Área de conocimiento de Didáctica y Organización Escolar, Facultad de Educación, Universidad de Murcia.España.monicavr@um.es

2 Universidad de Murcia. España. jesusmol@um.es

${ }^{3}$ Profesora de Educación Secundaria Obligatoria. España
} 
e sombras que ainda têm a Formação Profissional na Espanha, sendo este um momento importante depois da recente implantação da Formação Profissional Básica.

Formação profissional. Ciclo Médio. Reformas

\section{Introducción}

El estudio de la Formación Profesional (en adelante FP) ha sido un tema recurrente a lo largo del tiempo. En este sentido, son muchos los estudios publicados, aspectos analizados y campos científicos interesados por estas enseñanzas (Tejada, 2006; Bolívar y Gijón Puerta, 2008; Fasching, 2014; Gonon, 2014; González, González, Nieto, Portela, Porto y Vallejo, 2015, Jacob y Solga, 2015; Samanes, 2016), cuyo denominador común ha sido tratar de empoderar a la FP hacia el reconocimiento social que merece; en la medida en que se trata de una formación específica y cualificada que facilita a sus estudiantes las competencias académicas y profesionales necesarias para la adaptación a los cambios permanentes que exige la Sociedad y el mercado laboral. Esta encomiable finalidad ha quedado empañada tras una nube de desvalorización -e incluso de exclusión- que aún hoy no permite otear su potencial académico y formativo. Tal vez, la causa de esta desvaloración se encuentre en la tensión o falta de vínculo entre lo académico y lo profesional, y cuyo antecedente es el propio origen de las enseñanzas profesionales.

Cabe recordar que desde la Revolución Industrial (1780) hasta la primera mitad del pasado siglo (1950) se fueron consolidando diferentes modelos de formación que intentaban dar respuestas a las necesidades del mercado de trabajo, primando este ámbito por encima del académico. Estos modelos (sintetizados por Greinert, 2004) se conocen como el modelo liberal de mercado (de origen inglés), el modelo de regulación por el Estado (modelo francés), y el modelo corporativo y dual (modelo alemán). El modelo español podría situarse más cercano al modelo francés, aunque con algunos elementos propios del modelo alemán (modelo dual).

En base a todo ello, en el presente estudio se examina la evolución y la estructura de la FP, en España, haciendo un recorrido por sus diferentes leyes educativas. Se analiza la estructura organizativa, los criterios de acceso, los requisitos para migrar a otros estudios, el perfil docente requerido en las 
sucesivas etapas del sistema educativo; junto con una valoración final sobre las fortalezas y debilidades de dicha legislación.

El punto de partida será la Ley General de Educación de 1970, precisamente por tratarse de la primera ley que plantea un nuevo modelo de FP adaptado a las nuevas corrientes europeas de la época.

\section{Génesis y desarrollo de las reformas educativas sobre formación profesional}

Para indagar sobre los orígenes de enseñanzas con carácter profesionalizador desarrolladas en España, tendríamos que remontarnos a la Ley de Instrucción Pública (1857) -más conocida como Ley Moyano- ya las Escuelas Profesionales de Artes y Oficio creadas unos años más tarde (la primera de ellas se fundará en Sarriá, en 1886). Aunque no será hasta el año 1928, cuando se establezca un sistema reglado de enseñanzas profesionales y una red de centros que las desarrollen (Estatuto de Formación Profesional, 1928), siendo el responsable de aquellas enseñanzas el -entonces- Ministerio de Trabajo. Posteriormente, en el año 1955, ahora el Ministerio de Educación y Ciencia aprueba la Ley de Formación Profesional Industrial, que plantea como finalidad adecuar y actualizar el Estatuto de 1928, introduciendo profundas modificaciones, tanto en lo que respecta a los órganos rectores de la formación profesional, centros docentes y sistemas de enseñanza, como también en lo que atañe a la participación directa de la industria en la orientación y sostenimiento de esta importante faceta de la educación, que por primera vez se apoya sobre un fuerte soporte económico, resultado de la progresiva industrialización del país (Preámbulo, p. 4443).

Hasta estos momentos las enseñanzas profesionales tenían un marcado carácter industrial. Una concepción más amplia, con una estructura más sólida de la FP no se articulará hasta el año 1970, con la Ley General de Educación.

\subsection{La LGE: hacia una moderna Formación Profesional}

El paso de la Formación Profesional Industrial a la Ley General de Educación (1970) supuso una innovación importante en la concepción moderna de la enseñanza profesional, por cuanto el concepto clásico de oficio cedía paso 
a otros como profesión o familia profesional. La idea original pretendía establecer un tronco común y unificado con salidas profesionales al final de cada nivel.

\section{2 -Estructura y currículum}

Atendiendo a su estructura, la LGE (1970) plantea una FP organizada alrededor de tres grados, cada uno de los cuales no debía exceder los dos años de duración, simultaneándose el segundo año con prácticas formativas en empresas. Esta idea de entroncar la escuela con el entorno laboral será uno de los principios fundamentales sobre los que se quería construir una FP renovada. Tal innovación se dará de bruces con las limitaciones políticas y económicas de aquel periodo, impidiendo su materialización efectiva (Martínez Usarralde, 2002).

En relación al primero de los grados (FP1), indicar que estaba considerado como un instrumento para el desarrollo de la personalidad del alumno. Para acceder a esta etapa existía una doble vía: se podía acceder con el título de EGB - o certificado de escolaridad- o también (y en virtud de las disposiciones transitorias del Decreto 707/1976) se podría acceder acreditando el Título de Bachiller elemental, Certificado de Estudios Primarios o mediante una prueba de acceso (o curso de adaptación) para alumnos mayores de 14 años sin titulación (esta posibilidad se mantuvo hasta el curso académico 1979/1980).El plan de estudios de FP1 partía de un área formativa común, un área de ciencias aplicadas diversificada según la profesión, junto con un área de conocimientos teórico-prácticos de sus tareas propias (que incorporaba técnicas comunicativas y de expresión gráfica). El citado decreto establecía también que el área teóricopráctica debía tener un mínimo de mil horas lectivas, correspondiendo el último periodo con prácticas en empresas. De este modo, los alumnos que obtuviesen la titulación de primer grado serían Técnicos Auxiliares y a partir de dicha titulación, podrían acceder a Bachiller o a Enseñanzas profesionales especializadas.

El segundo grado (FP2) era considerado de mayor especialización; orientado hacia la preparación para el trabajo. Para acceder al mismo, los estudiantes debían tener el Título de Bachiller o haber cursado un primer grado de FP y enseñanzas complementarias (de un curso académico de duración). Los alumnos/as podrían quedar exentos de estas enseñanzas complementarias si 
demostraban madurez profesional específica. En este caso, las disposiciones transitorias del Decreto 707/1976 permitieron (hasta el curso académico 1979/1980) el acceso a bachilleres superiores -generales o técnicos, capataces agrícolas, instructores rurales, oficiales industriales y alumnos mayores de 16 años con titulaciones equivalentes. El plan de estudios de segundo grado tenía una doble vertiente: el régimen general y el de enseñanzas especializadas con carácter profesional. El régimen general consistía en un área formativa empresaria que contaba, además, con un área de conocimientos tecnológicos y prácticos -precisos para el ejercicio de la profesión-, así como técnicas de expresión gráfica y comunicación adecuadas que simultanearían (tras una fase inicial) con la práctica profesional. Además, incluía un área formativa común en idioma moderno, formación cívico-social y política, formación religiosa y educación físico-deportiva. Por otro lado, el régimen de enseñanzas especializadas era considerado como el instrumento de perfeccionamiento del segundo grado de FP. Estas enseñanzas consistían en una serie de fases sucesivas de ampliación de conocimientos con un área de formación básica. Cada fase tendría uno o más cursos complementarios (o parte de ellos) y en ningún caso superaría los tres años de duración. Al finalizar el régimen general (o el de enseñanzas especializadas), los alumnos/as obtendrían una titulación de Técnico Especialista con la que podrían acceder a centros universitarios en enseñanzas análogas a las cursadas.

El tercer grado (FP3) era considerado un complemento al primer ciclo universitario. Para acceder, los estudiantes debían acreditar estar en posesión del primer ciclo de una facultad o escuela técnica superior o -en su defectodebían poseer la titulación de Técnico especialista (FP2), así como determinadas enseñanzas complementarias (que dependerían -directamente- de la especialidad). Además, a este tercer grado pudieron acceder (hasta el curso académico 1979/1980) aquellos estudiantes universitarios con tres cursos aprobados, ingenieros, arquitectos técnicos, profesores mercantiles, asistentes sociales, graduados sociales, ayudantes técnicos sanitarios, maestros industriales, maestros de enseñanza primaria, profesores de enseñanza de hogar y aquellos a los que se refiere la disposición transitoria 11.2 de la LGE. Al 
finalizar estos estudios, el alumnado obtendría la titulación de Técnico Superior Diplomado y podrían acceder al segundo ciclo de una enseñanza universitaria de forma directa (o mediante las convalidaciones oportunas).

\subsection{El profesorado}

Los Centros de FP estarían dirigidos por un Director, seleccionado entre los profesores del Centro y nombrado por el MEC (toda vez oídos los órganos de Gobierno). Entre las funciones a desarrollar por el Director estarían precisamente- dirigir, orientar y coordinar todas las actividades del Centro y de sus órganos y -de modo especial-en lo referido al trabajo en equipo de sus profesores.

Con respecto al profesorado, la titulación que debían acreditar quedaba establecida en el artículo 102 de la ley, según el cual para ser profesor en FP1 se requería poseer un título de FP de segundo grado o enseñanzas especializadas. Para impartir docencia en segundo grado, los docentes debían acreditar una diplomatura, arquitectura técnica o ingeniería técnica, mientras que para ejercer la docencia en tercer grado sería necesario ser licenciado, arquitecto o ingeniero (además de poseer un certificado de especialización).

\subsection{Consideraciones generales}

Con esta ley se pretendió hacer frente a una notable contestación social, si bien sus impulsores no fueron capaces -finalmente- de forzar la previsión de mecanismos adecuados de financiación para implementar dicha reforma. Tal vez por ello es denominada como una criatura inadaptada a su tiempo (Martínez Usarralde, 2002). Además, el arranque en su aplicación coincidió con el inicio de la grave crisis económica de mediados de la década de los 70 .

Por otro lado, quedó patente -por la propia estructura organizativa y curricular que la FP era considerada una enseñanza de segunda categoría. Autores como Arroyo (1987) afirmaban que no debía servir para reproducir la división de clases sociales, poniendo de manifiesto la necesidad de ampliar los conciertos con los servicios públicos, las empresas públicas, instituciones y empresas privadas para la realización de las prácticas de los alumnos. La base de aquella argumentación se cimentada tras demostrar que aquellos países con 
mejores sistemas de formación sostenían una correspondencia con los menores índices de desempleo.

A pesar de todo, y como aspectos positivos a destacar, cabría reseñar la integración de la FP en la estructura del sistema educativo (educación general) y la germinación en la conciencia social de la necesidad de seguir formándose al término de la enseñanza obligatoria. Este fue el contexto sobre el que surgió la necesidad de una nueva reforma educativa, y el escenario para la promulgación de una nueva ley.

\section{La LOGSE: una ley de largo recorrido y mayor alcance}

La Ley Orgánica 1/1990 de Ordenación General del Sistema Educativo (LOGSE), señalaba en su preámbulo la necesidad de dar una correcta solución a los problemas estructurales y específicamente educativos, errores de concepción, insuficiencias y disfuncionalidades que se habían venido manifestando o agudizando con el paso del tiempo.

\subsection{Estructura y currículum}

El contenido de la FP se desarrolla en el RD 676/1993 donde se incluye la FP Superior se necesitaba el Título de bachillerato. Otros modos de acceso para estos ciclos, acabaron desarrollados en el RD777/1998, por el que se establecen determinados aspectos de la ordenación de la FP en el ámbito del sistema educativo. Asimismo, era posible acceder a través de una prueba regulada por las Administraciones educativas. En relación al currículum, el RD676/1993 establecía que los ciclos formativos se organizarían en módulos profesionales de formación teórico-práctica y su duración variaría en función de la naturaleza de la competencia profesional propia de cada título. Cada módulo de FP específica estaría asociado -además- a una o varias unidades de competencia y el currículo de los ciclos formativos incluiría un módulo de formación práctica en centros de trabajo (del que podrían quedar exentos los estudiantes que acreditasen experiencia laboral). La formación en centros de trabajo se desarrollaría en un ámbito productivo real en el que los alumnos/as podrían observar y desempeñar funciones propias de la profesión, conocer la organización de los procesos productivos o de los servicios reales, así como las 
relaciones socio-laborales en la empresa o en el centro de trabajo (Martínez Usarralde, 2002). Asimismo, la LOGSE explicitaba que para el diseño de los ciclos formativos se fomentaría la participación de agentes sociales. En su diseño, las enseñanzas mínimas incluían -para cada ciclo- objetivos generales, módulos profesionales necesarios para cada ciclo, duración total, objetivos (expresados en términos de capacidades terminales), contenidos de los módulos profesionales asociados a unidad de competencia, contenidos de los módulos profesionales de base (o transversales) y contenidos del módulo de FOL -que en ningún caso superarían el 55\% del horario previsto en comunidades con lengua cooficial (y el 65\% en comunidades sin lengua cooficial). También se contemplaba la posibilidad de ofertar las enseñanzas de FP específica a distancia y en otras modalidades horarias especiales adecuadas a las características de colectivos concretos, así como determinar las adaptaciones precisas respecto a criterios de admisión. de base y la específica. Esta primeraincluida en ESO y Bachillerato-estaría constituida por un conjunto de conocimientos, habilidades y destrezas comunes a un número amplio de técnicas o perfiles profesionales. La segunda, quedaría constituida por aquellos conocimientos, habilidades y destrezas vinculadas a la competencia profesional de cada título, y quedaba ordenada en ciclos formativos de Grado Medio y de Grado Superior, conducentes a la obtención de títulos profesionales. Para acceder al Grado Medio, los alumnos/as debían tener 18 años, haber completado la educación básica y acreditar el Graduado en Educación Secundaria (ESO). Y para el Grado

\subsection{El profesorado}

Según esta ley educativa se integraría en el cuerpo de profesores de enseñanza secundaria a los funcionarios pertenecientes -reglamentariamentea cuerpos de catedráticos, a los profesores agregados de Bachillerato y a los profesores numerarios de escuelas de maestría industrial. Asimismo, formarían parte del cuerpo de profesores técnicos de FP aquellos funcionarios del cuerpo de maestros de taller (de las escuelas de maestría industrial).

La regulación del acceso a este cuerpo quedaba establecida en el RD850/1993, donde se detalla el proceso de oposición a las especialidades; 
según éste, la selección de participantes se realizaría mediante tribunales y -en su caso- comisiones de selección u órganos equivalentes nombrados ad hoc. Para acceder al cuerpo de secundaria se requería el título de doctor, ingeniero, arquitecto, licenciado (o equivalente a efectos de docencia) además del título de especialización didáctica y la superación de un proceso selectivo. Para el acceso al cuerpo de profesores técnicos de FP, los aspirantes debían acreditar el título de arquitecto técnico, ingeniero técnico, diplomado universitario o equivalente, además del título de especialización didáctica y la superación de un proceso selectivo. Del mismo modo, para determinadas áreas o materias podrían demostrar la equivalencia a efectos de docencia de otras titulaciones.

En este texto normativo, se recogen también esas equivalencias a efectos de docencia y se establece su aplicación en los procedimientos selectivos que efectuasen las administraciones educativas en las tres primeras convocatorias de cada especialidad. Por otra parte, el llamado Certificado de Aptitud Pedagógica (CAP) sería el equivalente al título profesional de especialización didáctica (un curso de cualificación pedagógica -de un año de duración- con periodo de prácticas docentes). Quedarían exentos del mismo los maestros y licenciados en Pedagogía. Además, sería el Gobierno quien determinaría las circunstancias en que la experiencia previa se consideraría equivalente a este título profesional. En el caso de determinadas áreas o materias se contaría con profesores especialistas atendiendo tanto a su cualificación como a las necesidades del sistema. Este profesorado especialista también podría impartir clase -y de modo excepcional- en Bachillerato y únicamente en determinadas materias relacionadas con su experiencia profesional.

Por otra parte, el RD1635/1995 (por el que se adscribe el profesorado de los Cuerpos de Profesores de Enseñanza Secundaria y Profesores Técnicos de Formación Profesional a las especialidades propias de la FP específica), establecía las familias profesionales en las que quedaba organizada la FP específica, los ciclos formativos en ellas incluidos, las diversas especialidades de los cuerpos docentes, los módulos atribuidos a cada especialidad, así como la competencia docente en determinadas materias de Bachillerato (o la correspondencia entre antiguas y nuevas especialidades). Junto a ello, la 
LOGSE estableció-además- la formación permanente del profesorado como un derecho y una obligación, determinando que periódicamente el profesorado habría de realizar actividades de actualización científica, didáctica y profesional.

\subsection{Consideraciones generales}

Esta ley planteaba un cambio en el modelo pedagógico propuesto para esta etapa educativa, y sustentado -esencialmente en la definición del currículo por competencias profesionales junto con la implementación de una estructura formativa de carácter modular. Del mismo modo, la LOGSE establece una serie de acciones formativas que se llevan a cabo en el marco de la Formación Ocupacional y de la Formación Continua, y que si bien podría parecer que -en principio- se trata de una alusión de carácter meramente testimonial y que encuentra difícil acomodo en los Reales Decretos de desarrollo de la LOGSE, es posible afirmar que se trata de un valioso precedente a la hora de entender la evolución del modelo de FP impulsado en la legislación educativa española posterior (Ley Orgánica 5/2002, Ley Orgánica 10/2002, Ley Orgánica 2/2006 y Ley Orgánica 8/2013).

Si bien, y a pesar del aumento de la escolaridad obligatoria hasta los 16 años y la supresión de la doble titulación al finalizar la ESO, esta ley no consiguió eliminar los problemas atribuidos a la anterior FP1 (Cabrera, 1993). Esta reforma empezaría a mostrar sus efectos en el curso 1997/1998, en el que la FP de Grado Medio se ofertaba a todos los estudiantes que terminaban la etapa de secundaria. De igual forma, dejó sin resolver qué salidas eficaces ofrecer a aquella generación de jóvenes que abandonaron el sistema educativo pese a que debían permanecer dos años más en él (cabe recordar que la única propuesta fueron los Programas de Garantía Social que serían el precedente de los Programas de Iniciación Profesional -impulsados por la LOCE- y los ulteriores Programas de Cualificación Profesional Inicial -definidos en la LOE).

Como aspectos positivos cabría destacar la integración de la FP en los IES, un nuevo paso en relación a la LGE. Esta idea es el resultado del gran debate que se mantiene en la década de 1980 sobre la estructura de un tronco común que asegurase un enfoque global de las enseñanzas. Este aspecto supuso la ruptura entre la formación profesional de cualificación básica y la 
formación profesional de cualificación especializada. A pesar de todo ello, el lapso de tiempo entre el diseño de la ley y su implantación -junto con la crisis económica que vivió España después de 1992-pudo haber sido el condicionante de su exiguo éxito. Paradójicamente -y en palabras de Cabrera (1993)- se diseña en los ochenta para aplicarse en los noventa con el objeto de que funcione -a pleno rendimiento- en los primeros años del siglo XXI. A todas luces, se trata de un hándicap de partida para todas las enseñanzas regladas, y un obstáculo insalvable para la FP (García, Sales, Moliner y Ferrández, 2009).

\section{La LOCE: los Programas de Iniciación Profesional}

En el año 2002 llegaría la Ley Orgánica 10/2002 de Calidad de la Educación, no tanto para modificar la ordenación vigente en relación a la FP, sino más bien para otorgar de mayor flexibilidad al procedimiento de acceso a los ciclos. Los Programas de Iniciación Profesional (PIP) establecidos en la ley se conciben como una alternativa presidida por los principios de la máxima inclusión y la adecuada flexibilidad del sistema educativo, orientándose principalmente- a aquellos alumnos/as que rechazaban la escuela en su concepto tradicional. En esta ley (al igual que sucede con la Ley 2/2006 -LOE y la Ley 8/2013 -LOMCE) la FP reglada se estructura de acuerdo a las directrices de la Ley Orgánica 5/2002de las Cualificaciones y de la Formación Profesional, que establece la FP reglada como un subsistema. Se trata de un cambio conceptual relevante, por cuanto la FP ya no puede entenderse -únicamentecomo una etapa educativa, sino como algo que forma parte de un sistema más complejo, debiendo compartir y coordinar sus propuestas con las que se promueven en otros ámbitos (subsistema de FP para el empleo, procedimientos de acreditación de competencias profesionales, etc.). Sin lugar a dudas, se trata de aportes significativos para comprender la evolución de la FP en España durante los últimos años.

\subsection{Estructura y currículum}

Los PIP estaban integrados por los contenidos curriculares esenciales de la formación básica y por módulos profesionales asociados -al menos- a una cualificación del Catálogo Nacional de Cualificaciones Profesionales a que se 
refiere el artículo 7 de la citada ley. Dicha formación, que tendría una estructura flexible de carácter modular, sería impartida en dos cursos académicos, siendo el Gobierno el responsable de fijar las directrices básicas de estos programas. Así, aquellos estudiantes que -cumplidos los 15 años y tras la adecuada orientación educativa y profesional- optasen voluntariamente por no cursar ninguno de los itinerarios ofrecidos con carácter general, quedarían escolarizados en estos programas, a los que también podrían incorporarse alumnos con 16 años cumplidos.

La superación del PIP daría derecho a la obtención del título de Educación Secundaria Obligatoria (ESO), mientras que la superación total o parcial de los módulos de carácter profesional integrados en los PIP sería acreditada conforme a lo establecido en el artículo 8 de la citada ley de Cualificaciones y de la Formación Profesional. Posteriormente, el RD 362/2004 (por el que se establece la ordenación general de la FP específica) establecería aspectos comunes para la FP que incluirían: módulos formativos relacionados con la orientación laboral y las relaciones laborales (dirigidos a conocer las oportunidades de aprendizaje y acceso o reinserción laboral); módulos de prevención de riesgos laborales (que incorporarían enseñanzas necesarias para la prevención de los riesgos derivados del ejercicio profesional correspondiente); enseñanzas relacionadas con las tecnologías de la información y la comunicación (relativas al ámbito profesional correspondiente); un módulo de enseñanzas de una lengua de los estados miembros de la Unión Europea y enseñanzas orientadas a fomentar el espíritu emprendedor.

\subsection{El profesorado}

Para impartir estas enseñanzas se requería el título de Doctor, Licenciado, Ingeniero, Arquitecto o equivalente a efectos de docencia, mientras que en aquellas asignaturas que determinasen su relación con la FP se establecerían equivalencias de ingeniero técnico, arquitecto técnico y diplomado (además, sería necesario estar en posesión del título de Especialización Didáctica). Por otra parte, para impartir módulos profesionales integrados en los PIP se podría contratar como profesores especialistas -atendiendo a su cualificación y necesidades del sistema- a profesionales que desarrollasen su 
actividad en el ámbito laboral. Además -tal como ya se ha señalado anteriormente- el procedimiento de acceso a ciclos era más flexible; como norma general podrían cursar FP de Grado Medio quienes estuvieran en posesión del título de Graduado en Educación Secundaria Obligatoria. Para cursar FP de Grado Superior, los alumnos/as debían poseer el título de Bachiller, pudiendo también acceder a la FP aquellos aspirantes que -careciendo de los requisitos académicos- superasen una prueba de acceso. Para acceder mediante esta vía a los ciclos formativos de Grado Superior se requeriría tener 20 años de edad (cumplidos en el año de realización de la prueba).

Para realizar la prueba de acceso a Grado Medio, los alumnos/as quedarían exentos de acreditar las capacidades profesionales si hubieran superado la totalidad de módulos profesionales de un PIP, o si pudieran acreditar experiencia laboral relacionada con la enseñanza a cursar. Para el acceso a Grado Superior, los alumnos quedarían exentos de justificar las capacidades profesionales acreditando experiencia laboral relacionada con los estudios.

Del mismo modo, los estudiantes que hubieran cursado Bachillerato podrían acceder a los ciclos de Grado Superior mediante una prueba que acreditase las capacidades del alumno en relación al campo profesional al que pretendiesen acceder. En el caso de aquellos alumnos/as que estuvieran en posesión del Título de Técnico, y que deseasen acceder a un ciclo formativo superior relacionado con el mismo, sería prescriptivo acreditar la madurez en relación a los objetivos de Bachillerato; para tales alumnos/as, el requisito de edad para la realización de la prueba sería de 18 años cumplidos en el año natural.

\subsection{Consideraciones generales}

La victoria electoral del partido socialista, en marzo de 2004, paralizó frontalmente la LOCE y supuso el inicio de un nuevo y largo proceso de debate sobre la situación del sistema educativo. El fracaso y abandono escolar seguía siendo una realidad palmaria, en la que el 25\% del alumnado fracasaba en sus estudios o no lograba una titulación al finalizar la Educación Secundaria Obligatoria (ESO). Tan confusa llegó a ser la situación, que se hablaba -incluso- 
de la irresolución de los problemas educativos a través de la aplicación de reformas como instrumento de cambio eficaz (Sarason, 2003).

\section{La LOE: una apuesta hacia la nueva concepción de la Formación Profesional}

Tras un breve periodo de tiempo desde la implantación de la LOCE, el 03 de mayo de 2006 fue publicada la Ley Orgánica de Educación (LOE). En ella se encierra una división de la educación secundaria articulada a partir de una etapa obligatoria (ESO), seguida de la Educación Secundaria Postobligatoria (Bachillerato, FP de grado medio, Enseñanzas profesionales de Artes plásticas y diseño -de grado medio- y Enseñanzas deportivas -también de grado medio); formando parte la FP de Grado Superior de la Educación Superior.

\subsection{Estructura y currículum}

Con la LOE, entran en vigor los Programas de Cualificación Profesional Inicial (PCPI), donde-excepcionalmente- se anticipa el acceso a los alumnos/as de 15 años que habiendo cursado segundo no están en condiciones de promocionar a tercero -ya han repetido una vez en secundaria. Para ellos se ofertan los módulos que conducen a la obtención del título de Graduado en Educación Secundaria Obligatoria. Los PCPI incluirían tres tipos de módulos: módulos específicos referidos a las unidades de competencia correspondientes a cualificaciones de nivel uno (del Catálogo Nacional de Cualificaciones Profesionales); módulos formativos de carácter general (que amplían competencias básicas y favorecen la transición desde el sistema educativo al mundo laboral), y los módulos de carácter voluntario para los alumnos/as (que conducen a la obtención del título de Graduado en ESO y que podrían cursarse de manera simultánea con los anteriores, o una vez superados estos). Los ciclos formativos serían de Grado Medio y de Grado Superior, estando referidos al citado catálogo, constituyendo -respectivamente- la FP de Grado Medio y la FP de Grado Superior.

Paralelamente, se establece que los estudios de FP podrán realizarse tanto en centros educativos como en centros integrados de referencia nacional. Este hecho constituye una novedad importante, ya que estos centros integrados 
tienen implantación en todas las comunidades autónomas del estado español y están especializados en los diferentes sectores productivos. A tales efectos, estos centros podrían incluir acciones formativas dirigidas a estudiantes, trabajadores en activo y desempleados, así como a empresarios y formadores.

Con relación al acceso, los requisitos generales para acceder a Grado Medio (título de Graduado en ESO) y Grado Superior (título de Bachiller) serían los mismos que en las anteriores leyes (LOGSE y LOCE). Sin embargo, la LOE establece modificaciones en las pruebas de acceso reguladas por las administraciones educativas; para acceder mediante esta vía a ciclos formativos de Grado Medio, sería necesario tener 17 años -como mínimo- y en el caso de Grado Superior la edad establecida sería 19 años (en ambos casos debían ser cumplidos en el año de realización de la prueba).

Al igual que en normativas anteriores, el currículo de las enseñanzas de FP incluía una fase de formación práctica en los centros de trabajo. La superación del ciclo formativo requeriría de una evaluación positiva en todos los módulos que lo componen. De este modo, los alumnos/as que superasen las enseñanzas de FP de Grado Medio recibirían el Título de Técnico de la correspondiente profesión, el cual permitiría el acceso directo a todas las modalidades de Bachillerato. Por su parte, los alumnos/as que superasen las enseñanzas profesionales de Grado Superior obtendrían el Título de Técnico Superior. Esta titulación les daría acceso a los estudios universitarios que determinase el Gobierno, previa consulta a las comunidades autónomas e informe del Consejo de Coordinación Universitaria. Además, aquellos estudiantes que no hubieran superado la totalidad de las enseñanzas de cada ciclo formativo recibirían un certificado académico de los módulos superados con efectos de acreditación parcial y acumulable de las competencias en relación con el Sistema Nacional de Cualificaciones y Formación Profesional.

\subsection{El profesorado}

Para impartir docencia en estos programas, la LOE exige -al igual que en Educación Secundaria Obligatoria- el título de Licenciado, Ingeniero o Arquitecto (o título de Grado equivalente), además de la formación pedagógica y didáctica de nivel de postgrado. No obstante, serían consideradas equivalentes a la 
formación exigida los títulos Profesionales de Especialización Didáctica y el Certificado de Cualificación Pedagógica (que a la entrada en vigor de esta ley hubieran organizado las universidades al amparo de lo establecido en las anteriores leyes orgánicas), junto con el Certificado de Aptitud Pedagógica y otras certificaciones planteadas por el Gobierno, quedando exentos de esta exigencia los maestros y licenciados en Pedagogía y Psicopedagogía, así como quienes estuviesen en posesión de licenciatura o titulación equivalente que incluyese formación pedagógica y didáctica. Excepcionalmente, la ley contemplaba que para determinados módulos se podría incorporar -como profesores especialistas- a profesionales no necesariamente titulados que desarrollasen su actividad en el ámbito laboral -en régimen laboral o administrativo- y de acuerdo con la normativa resultante de aplicación.

Asimismo, se establecía que los profesores técnicos que a la entrada en vigor de esta ley estuvieran impartiendo docencia en Bachiller, podrían continuar de forma indefinida en esta situación. Para el ingreso al cuerpo de profesores de enseñanza secundaria sería necesario estar en posesión del título de Doctor, Licenciado, Ingeniero, Arquitecto (o el título de Grado correspondiente u otros títulos equivalentes a efectos de docencia), además de la formación pedagógica y didáctica y la superación del proceso selectivo. Para el ingreso en el cuerpo de profesores técnicos de FP sería necesario estar en posesión de la titulación de Diplomado, Arquitecto Técnico, Ingeniero Técnico (o el título de Grado correspondiente u otros títulos equivalentes a efectos de docencia), además de la formación pedagógica y didáctica y la superación del proceso selectivo. En este caso, podía exigirse también a los profesores técnicos de Formación Profesional una experiencia profesional en un campo laboral relacionado con la materia o área a la que se optaba.

\subsection{Consideraciones generales}

De alguna manera esta ley comienza a establecer las bases para una formación profesional reglada que apuesta por una educación de calidad en el amplio sentido de la palabra; una educación que permita a la sociedad ser competitiva en el ámbito socio-económico; lo que implica concebir una formación profesional de calidad. De hecho, es esta idea de competitividad la que sustenta 
la aprobación de la Ley de Economía Sostenible (Ley 2/2011), que persigue facilitar la adecuación de la oferta formativa a las demandas del sistema productivo, ampliar la oferta de formación profesional, avanzar en la integración de la formación profesional en el conjunto del sistema educativo y reforzar la cooperación de las administraciones educativas (p.25047). Esta ley (aprobada en plena crisis económica) no tiene una gran continuidad legislativa y se ve superada por una nueva -y reciente- reforma.

\section{La LOMCE: ¿La última reforma educativa?}

La estrenada Ley Orgánica para la Mejora de la Calidad Educativa (LOMCE, 2013) entra en vigor no sin una gran controversia y oposición social. Tal y como reza en su preámbulo, se trata de un ordenamiento que propone la modernización de la oferta formativa, su adaptación a los requerimientos de los diferentes sectores productivos, la implicación de las empresas en el proceso formativo, junto con la importante novedad de la Formación Profesional dual, y la búsqueda de un acercamiento a los modelos de los países del entorno con niveles mucho menores de desempleo juvenil (p.97865).

\subsection{Estructura y currículum}

Con esta ley se crea un nuevo título de FP básica de carácter obligatorio y gratuito. La FP comprende ahora los ciclos de Formación Profesional Básica, de Grado Medio y de Grado Superior, con una organización modular -de duración variable- que integra los contenidos teórico-prácticos propios de los diversos campos profesionales.

El currículo de las enseñanzas de FP incluye una fase de formación práctica en los centros de trabajo, de la que podrán quedar exentos quienes acrediten una experiencia laboral correspondiente con los estudios cursados. Asimismo, los ciclos de FP Básica (regulados en el RD127/2014) ordenan la adquisición de las competencias del aprendizaje permanente a través de la impartición de enseñanzas organizadas por los siguientes bloques comunes: Bloque de Comunicación y Ciencias Sociales (que incluirá las materias de lengua castellana, lengua extranjera, ciencias sociales y -en su caso- lengua cooficial) y Bloque de Ciencias Aplicadas (que incluirá matemáticas aplicadas al contexto 
personal y de aprendizaje en un campo profesional y ciencias aplicadas al contexto personal y de aprendizaje en un campo profesional). Además, las enseñanzas de FP Básica garantizarán -según este real decreto- la formación necesaria para obtener una cualificación de nivel 1 del Catálogo Nacional de las Cualificaciones Profesionales. Se trata de ciclos que tendrán una duración de 2000 horas, equivalentes a dos cursos académicos a tiempo completo y en los que los alumnos/as podrán permanecer durante un máximo de cuatro años.

Del mismo modo -y en el marco de lo establecido por las administraciones educativas- los centros educativos podrán ofertar al alumnado que curse ciclos formativos de Grado Medio las siguientes materias voluntarias para facilitarles la transición a otras enseñanzas: comunicación en lengua castellana, comunicación en lengua extranjera, matemáticas aplicadas (y -en su casocomunicación en lengua cooficial). Además, y con objeto de facilitar la progresión a ciclos formativos de Grado Superior, los centros educativos podrán ofertar materias voluntarias relacionadas con el campo o sector profesional del que se trate, de forma presencial o también a distancia. Esta posibilidad se asienta en el reconocimiento de la existencia de un dinamismo profesional, hecho que exige una continua evolución de las cualificaciones y competencias, debido esencialmente a la informatización y automatización de una serie de tareas, lo que repercute en la organización, logística y en la gestión del trabajo. Por este motivo, la modalidad a distancia comparte así objetivos comunes con la enseñanza presencial, no entendiéndose ya como subsidiaria de ésta.

En cuanto al acceso a los ciclos de FP Básica, la LOMCE establece el requerimiento del cumplimiento simultáneo de las siguientes condiciones: tener cumplidos 15 años -o cumplirlos durante el año natural en curso- y no superar los 17 años de edad en el momento del acceso (o durante el año natural en curso); haber cursado el primer ciclo de ESO (o excepcionalmente haber cursado el segundo curso de la ESO y haber propuesto el equipo docente a los padres o tutores- la incorporación del estudiante a un ciclo de Formación Profesional Básica). Para el acceso a ciclos formativos de Grado Medio se deberá cumplir al menos- una de las siguientes condiciones: estar en posesión de uno de los siguientes títulos: Título de Graduado en ESO (siempre que el alumno haya 
superado la evaluación final de esta etapa por la opción de enseñanzas aplicadas), Título Profesional Básico, Título de Bachiller, un título universitario, un título de Técnico o Técnico Superior de Formación profesional, estar en posesión de un certificado acreditativo de haber superado todas las materias de Bachillerato (o haber superado un curso de formación para acceso a ciclos de grado medio en centros autorizados por la administración educativa y tener 17 años cumplidos en el año de realización de dicha prueba). Y, para acceder a Grado Superior, será necesario reunir uno de los siguientes requisitos: estar en posesión del título de Bachiller, de un título universitario, de un título de Técnico o de Técnico Superior de Formación Profesional, de un certificado acreditativo de haber superado todas las materias de Bachillerato, haber superado una prueba de acceso -de acuerdo con los criterios establecidos por el Gobierno- y tener 19 años cumplidos en el año de realización de dicha prueba.

Vinculado a lo expuesto, otra novedad -no exenta de polémica-, es que los padres-tutores, o incluso los propios estudiantes, podrán escoger cursar el cuarto curso de la ESO con la opción de enseñanzas aplicadas para la iniciación a la FP o con la opción de enseñanzas aplicadas para la iniciación al Bachillerato.

El currículum de estas enseñanzas se ajustará a las exigencias derivadas del Sistema Nacional de Cualificaciones y Formación Profesional, siendo responsabilidad del Gobierno el desarrollo de correspondencias y equivalencias con certificados de profesionalidad, a través de unidades de competencia acreditadas. Estos certificados de profesionalidad serán el instrumento de acreditación oficial de las cualificaciones profesionales en el ámbito de la administración laboral, en virtud de las cuales se acredita la capacitación para el desarrollo de una actividad laboral con significación para el empleo, y asegurando la formación necesaria para su adquisición desde el marco del subsistema de formación profesional para el empleo. Se configura así un perfil profesional entendido como un conjunto de competencias profesionales identificable en el sistema productivo, reconocido y valorado en el mercado laboral (RD 34/2008, p. 5685). Estos certificados están regulados por el RD 34/2008 (a su vez modificado por el RD 189/2013), a fin de adecuar la regulación de los certificados de profesionalidad en relación con el nuevo contrato para la 
formación y el aprendizaje (regulado en el RD 1529/20112, en el que también se establecen las bases de la formación profesional dual).

En cuanto a la evaluación del aprendizaje del alumnado, en todos los ciclos formativos este proceso se realizará por módulos profesionales y, en su caso, por materias o bloques. Desde esta óptica, los alumnos/as que superen un ciclo de FP Básica recibirán el título Profesional Básico correspondiente; el título Profesional Básico permitirá el acceso a los ciclos formativos de Grado Medio de la FP del sistema educativo. Los estudiantes podrán obtener el título de Graduado en Educación Secundaria Obligatoria por cualquiera de las dos opciones de evaluación final que establece la ley. Además, cuando una persona mayor de 22 años tenga acreditadas unidades de competencia de un título profesional básico -mediante un Certificado de profesionalidad de nivel 1 o por el procedimiento de evaluación y acreditación establecido- recibirá de las administraciones el Título Profesional Básico. Del mismo modo, los alumnos/as que superen los ciclos formativos de Grado Medio recibirán el Título de Técnico de la correspondiente profesión. Con el título de Técnico -y mediante un procedimiento de admisión- podrán acceder a Grado Superior.

\subsection{El profesorado}

Una novedad importante de la actual legislación en materia de profesorado, es que ahora se establece que los miembros del equipo directivo y los profesores serán considerados autoridad pública. Por ello, en los procedimientos de adopción de medidas correctoras, los hechos constatados por el profesorado de los centros docentes tendrán valor probatorio y disfrutarán de presunción de veracidad "iuris tantum" (Art. 124, p. 97903). De este modo, los docentes obtienen un respaldo legal que ya había sido incorporado en normativas autonómicas de diversas comunidades, y que venía siendo reivindicado desde hacía ya varios años por el colectivo docente. Por último, y con respecto a las titulaciones exigidas al profesorado de Formación Profesional y Enseñanza Secundaria y a la ordenación de los cuerpos docentes, la presente ley no incluye ninguna modificación respecto a la ley anterior. 


\subsection{Consideraciones generales}

La necesidad de nueva ley educativa se sustenta -gubernamentalmenteen los malos resultados obtenidos en diferentes indicadores nacionales y/o internacionales (PISA, Eurostat, EPA, Panorama de la Educación, 2012). Indicadores que ponen de manifiesto los bajos resultados académicos, el alto porcentaje de abandono escolar temprano o las elevadas tasas de paro juvenil en España (Catterall, Davis y Yang, 2014). A pesar de estas evidencias- muchos colectivos plantean dudas sobre la fiabilidad de esta ley para subsanar tales problemáticas, o si -por el contrario- será un nuevo desaliento para la dignificación de la FP; máxime cuando se gesta, discute y finalmente se aprueba sin ningún tipo de consenso parlamentario.

Lo que sí parece evidente es que esta falta de consenso y la celeridad en la implantación de esta reforma no parecen ser el mejor contexto -ni el más coherente- para la actual realidad educativa.

\section{Conclusiones}

Durante un largo periodo de tiempo -anterior a la entrada en vigor de la Ley General de Educación (1970)- las enseñanzas profesionales, en España, estuvieron divididos en dos niveles: Oficialía Industrial y Maestría Industrial. Con la promulgación de la LGE (1970), la FP quedó conformada en dos niveles que se denominaron grados. Así, aparecieron la FP1 y la FP2. Posteriormente, y con la entrada en vigor de la LOGSE, la legislación educativa establece la diferenciación entre FP de base y FP específica, estando la primera integrada en los ciclos obligatorios (Primaria y ESO) y formando parte del currículum general; mientras que la segunda la constituirían los ciclos formativos de Grado Medio y Superior -sin conexiones ni pasarelas-y dando lugar a una formación insuficiente dentro de un campo profesional limitado para encarar la transición a la vida activa. Años más tarde, la LOE vendría a modificar ciertas condiciones de acceso e incluso determinaría una reserva de plazas para alumnos con discapacidad. Sin embargo -y a pesar de los esfuerzos por evitarlo- la LOMCE fue publicada y con ella ha llegado la FP Básica y la FP dual como principales novedades. 
Estas dos décadas de reformas y contrarreformas -por las que ha transitado la FP- no han permitido solventar esa tensión existente entre lo académico y lo profesional, al igual que tampoco ha permitido disipar esa nube de desvalorización que parece acompañarla. Realidad que se hace más preocupante, si cabe, con la última reforma educativa. A saber, aquellos alumnos/as que superen un ciclo de FP básica recibirán el título de formación profesional Básica pese a que ello no les permitirá obtener -de forma automáticael Título de la ESO; debiendo realizar una prueba específica. Así, esta formación pareciera perder ahora su funcionalidad como recurso educativo, convirtiéndose en un itinerario de segunda categoría que aglutinaría -precisamente- a aquellos estudiantes con peor rendimiento académico y mayor (de la mal llamada) inadaptación al medio social; es decir, que mostrando una ablepsia total hacia los propios principios recogidos en la ley (referidos a calidad, equidad, no discriminación, igualdad de oportunidades -Artículo 2), y sin ser la FP Básica una medida de atención a la diversidad (explícita), se selecciona y separa a los alumnos/as consiguiendo así que todo el 4º curso de la ESO sea un "garante de éxito".

Sin lugar a dudas, y solo tal vez, hay algo de premeditación en estas palabras considerando que éste es el primer año de la implantación de la LOMCE. Si bien, lo que sí parece del todo razonable es suponer que de nada nos servirá ejecutar leyes sin constatar verdaderamente sus fortalezas y carencias; en definitiva, sin evaluarlas antes de derogarlas. Este ha sido el pequeño ejercicio de reflexión que hemos querido hacer con el presente trabajo.

\section{Referências}

Arroyo, S. (1987). Relación entre Formación Profesional reglada y ocupacional. Seminario sobre el Proyecto para la Reforma de la Enseñanza. Madrid: Consejo Escolar del Estado.

Bolívar, A. y Gijón Puerta, J. (2008). Historias de vida que deshacen profecías de fracaso. Cuadernos de Pedagogía, 382, 56-59.

Cabrera, L. C. (1993). La tortuosa marcha de la formación profesional en España. Revista de educación, $302,193-212$.

Catterall, J., Davis, J. y Yang, D. (2014). Facilitating the learning journey from vocational education and training to higher education. Higher Education Research \& Development, 33(2), 242-255. 
Decreto 707/1976, de 5 de marzo, sobre Ordenación de la Formación Profesional (BOE no 88, de 12 de abril).

Fasching, H. (2014). Intellectual disability and gender in the transition from school to vocational training and employment. Journal of Applied Research in Intellectual Disabilities, 27(4), 373-378.

García, R., Sales, A., Moliner, O. y Ferrández R. (2009). La formación ética profesional desde la perspectiva del profesorado universitario. Teoría de la Educación, 21(1), 199-221.

Gonon, P. (2014). Meta-Evaluation Training Objects, Effects and Success Factors for German Vocational Training Cooperation. Zeitschrift fur evaluation, 13(1), 146-148.

González, MT., González, EM, Nieto, JM, Portela, A., Porto, M y Vallejo, M. (2015). La vulnerabilidad escolar y los programas de Cualificación Profesional Inicial: apuntes para la formación profesional básica. Madrid: Wolters Kluwer.

Greinet, W. D. (2004). Los sistemas europeos de formación profesional: algunas reflexiones sobre el contexto teórico de su evolución histórica. Revista de Formación Profesional, 32, 18-26.

Jacob, M. y Solga, H. (2015). Germany's Vocational Education and Training System in Transformation: Changes in the Participation of Low-and High-Achieving Youth Over Time. European Sociological Review, 31(2), 161-171.

Ley de Instrucción Pública de 09 de septiembre de 1857 (Ley Moyano).

Ley de 20 de julio de 1955, sobre la Formación Profesional Industrial. BOE no 202, de 21 de julio.

Ley 14/1970, de 4 de agosto, General de Educación y Financiamiento de la Reforma Educativa. BOE ํㅜ 187, de 6 de agosto.

Ley Orgánica 1/1990, de 3 de octubre, de Ordenación General del Sistema Educativo. BOE nำ238, de 4 de octubre.

Ley Orgánica 5/2002, de 19 de junio, de las Cualificaciones y de la Formación Profesional. BOE no 147, de 20 de junio.

Ley Orgánica 10 /2002, de 23 de diciembre, de Calidad de la Educación. BOE no 307, 24 de diciembre.

Ley Orgánica 2/2006, de 3 de mayo, de Educación. BOE ํㅜ 106, de 4 de mayo.

Ley $2 / 2011$, de 4 de marzo, de Economía Sostenible. BOE nํ5, de 5 de marzo.

Ley Orgánica 8/2013, de 9 de diciembre, para la mejora de la calidad educativa. BOE no 295 , de 10 de diciembre.

Martínez Usarralde, M. J. (2002). Historia de la formación Profesional en España. De la ley de 1955 a los Programas Nacionales de Formación Profesional. Valencia: Universidad de Valencia.

Real Decreto Ley de 21 de diciembre de 1928: Estatuto de Formación Profesional.

Real Decreto 676/1993, de 7 de mayo, por el que se establecen directrices generales sobre los títulos y las correspondientes enseñanzas mínimas de formación profesional. BOE ํㅜ 122, de 22 de mayo.

Real Decreto 850/1993, de 4 de junio, por el que se regula el ingreso y la adquisición de especialidades en los Cuerpos de funcionarios Docentes a que se refiere la Ley 
Orgánica 1/1990, de 3 de octubre, de Ordenación General del Sistema Educativo. BOE no 155 , de 30 de junio.

Real Decreto 1635/1995, de 6 de octubre, por el que se adscribe el profesorado de los Cuerpos de Profesores de Enseñanza Secundaria y Profesores Técnicos de Formación Profesional a las especialidades propias de la formación profesional específica. BOE no 242, de 10 de octubre.

Real Decreto 777/1998, de 30 de abril, por el que se desarrollan determinados aspectos de la ordenación de la formación profesional en el ámbito del sistema educativo. BOE $n^{\circ} \mathbf{1 1 0}$, de 08 de mayo.

Real Decreto 362/2004, de 5 de marzo, por el que se establece la ordenación general de la formación profesional específica. BOE no 74, de 26 de marzo.

Real Decreto 34/2008, de 18 de enero, por el que se regulan los certificados de profesionalidad. BOE no 27 , de 31 de enero.

Real Decreto 1529/2012, de 8 de noviembre, por el que se desarrolla el contrato para la formación y el aprendizaje y se establecen las bases de la formación profesional dual. BOE $\mathrm{n}^{\mathrm{0}} \mathbf{2 7 0}$, de 9 de noviembre.

Real Decreto 189/2013, de 15 de marzo, por el que se modifica el Real Decreto 34/ 2008, de 18 de enero, por el que se regulan los certificados de profesionalidad y los reales decretos por los que se establecen certificados de profesionalidad dictados en su aplicación. BOE no 69, de 21 de marzo.

Real Decreto 127/2014, de 28 de febrero, por el que se regulan aspectos específicos de la Formación Profesional Básica de las enseñanzas de formación profesional del sistema educativo, se aprueban catorce títulos profesionales básicos, se fijan sus currículos básicos y se modifica el Real decreto 1850/2009, de 4 de diciembre, sobre expedición de títulos académicos y profesionales correspondientes a las enseñanzas establecidas en la Ley Orgánica 2/2006, de 3 de mayo, de Educación. BOE nº 55, de 05 de marzo.

Samanes, B. E. (2016). Transferencia del sistema de FP dual a España. Revista de Investigación Educativa, 34(2), 295-314.

Saranson, S. (2003). El predecible fracaso de la reforma educativa. Barcelona: Octaedro.

Tejada, J. (2006). Elementos de convergencia entre la formación profesional y la universidad: implicaciones para la calidad de la formación profesional superior.

Revista de Educación, 340, 1085-1117. 
Evolución de la formación profesional en España:

medio siglo de cambios y reformas

Interritórios | Revista de Educação Universidade Federal de Perna mbuco Caruaru, BRASIL | V.3 | N.4 [2017] 